\title{
Operaciones vinculadas y rentabilidad de la empresa A. Hatrodt Perú S.A.C, 2016-2018
}

\section{Related transactions and the profitability of the company A. Hartrodt Peru S.A.C, 2016-2018}

Mirian Yanina Segura Tapia

ORCID: 0000-0002-8088-7785

Universidad Norbert Wiener, Perú

Autor para correspondencia: mirian.segura27@gmail.com

Fecha de recepción: 16 de junio del 2019 - Fecha de aceptación: 23 de agosto del 2019

Resumen: El siguiente artículo es de naturaleza descriptiva y tiene por objetivos, analizar las operaciones vinculadas y la rentabilidad económica y financiera de la empresa A. Hartrodt. Las empresas están en constante adaptación a las diversas necesidades del mercado, es por ello que surgen estrategias claves para expandirse e incrementar las ganancias; de esta manera existen grupos empresariales que han surgido rigiéndose bajo una matriz y sucursales en diferentes mercados del mundo. El enfoque que se utilizó es de origen cuantitativo, el diseño y método utilizado fue descriptivo; se debe agregar que para la compilación de datos se utilizó los saldos de las cuentas de los estados financieros de tres periodos contables; y el método de análisis de datos fue la aplicación de los ratios de rentabilidad. Los resultados señalan que las operaciones vinculadas y la rentabilidad de la compañía, tienen incidencias en los resultados de los periodos debido a que sus operaciones a nivel internacional lo realizan con las intercompañías, lo que permite mayor fluidez en el proceso de las operaciones y optimizan los recursos. No obstante, el tener tanta facilidad para ejecutar sus transacciones, puede repercutir en la omisión de un análisis a profundidad que permita identificar si son efectivamente rentables o no.

Palabras claves: operaciones vinculadas; rentabilidad; estados financieros; ratios financieros; impuestos

Abstract: The following article is descriptive and aims to analyze the vinculated operations and the economic and financial profitability of the company A. Hartrodt. Nowadays, the companies are constantly adapting to the diverse business necessities, which is why they develop key strategies to expand and increase its profits. In this way, there are business groups that have emerged as linked companies and subsidiaries in different markets around the world. The approach that was used is quantitative, the design and method used were descriptive. It should be noted that the balances of the financial statements of the three years analyzed were used for the compilation of data; and the method of data analysis was the application of profitability ratios. The results indicate that the linked operations have incidences in the company due to the fact that allows greater fluidity in the process of the operations and optimizes the resources. However, having so much facility to execute their transactions may drift in the omission of an in-depth analysis that allows to identify if these kind of operations are effectively profitable or not.

Key Words: related operations; profitability; financial statements; financial ratios; taxes 


\section{Introducción}

En el último siglo, la globalización ha impactado potencialmente en las actividades que se desarrollan a nivel tecnológico, social, ambiental, cultural y en mayor medida en el aspecto económico empresarial, porque se han generado cambios y se demanda que las empresas sean más competitivas, brinden servicios, productos y bienes de mejor calidad y con ello alcancen la especialización en su giro de negocio para mantenerse en el mercado. Más aún, lo que se busca es fomentar el crecimiento responsable y crear bienestar entre las entidades de los diferentes grupos económicos.

A causa de estos grandes cambios se creó en 1961 la Organización para la Cooperación y el Desarrollo Económico, organismo que tiene directrices sobre las transacciones económicas de carácter vinculado.

En el Perú la legislación para el Estudio de Precios de Transferencia (EPT) se da desde el 01 de enero del año 2001. Sin embargo, desde el año 2006 entra en vigencia su aplicación de carácter obligatorio para aquellas empresas que realicen operaciones con vinculación económica acorde al Reglamento de la Ley de Impuesto a la Renta. A causa de ello, los profesionales en contabilidad han tomado nuevas perspectivas para el desarrollo y tratamiento de tales operaciones que son reflejadas en los estados financieros (Rueda, 2017).

Von B., sustentó bajo el enfoque de la organización como sistema abierto sobre a las entidades, quienes son fundadas y creadas por el ser humano, el cual está en constante relación con el ambiente que lo rodea; y tienen como finalidad obtener beneficios económicos futuros a través de sus operaciones empresariales, para ello de manera inherente se relaciona con otros entes de personalidad jurídica o natural, así como también clientes internos (Chiavenato, 2006).

Por otro lado, la teoría de responsabilidad social en el ámbito empresarial se traduce en el deber que se genera entre los participantes que forman la cadena de valor de una compañía, porque obedecen a una planificación previamente evaluada y aprobada (D’Onofrio, 2006). Las perspectivas que se vienen aplicando de manera tradicional están enfocadas en los capitales aportados y en el impacto de los índices financieros obtenidos de los saldos de las cuentas contables, solo manifiestan parte de la trayectoria (Daft, 1989). Finalmente, la teoría jurídica estudia la naturaleza de las sociedades y está conformado por un grupo mayor igual a dos socios o accionistas, abe resaltar, que el patrimonio de la organización versus el de los socios tienen el principio de separación (Junyent y Richard, 2009).

Una compañía se encarga de establecer diversos procedimientos a través del personal de la misma para poder llevar a cabo su actividad económica, incurriendo en operaciones financieras, utilizando capital de trabajo y con ello alcanzar sus propósitos (Fernández, Llorente y Pérez, 2008). Además, la empresa es un conglomerado de competencias y considera al talento humano para complementar y aprovechar los conocimientos y destrezas con la finalidad de crear o fomentar mayor valor al bien o servicio ofertado (Tilton, 1962).

Las operaciones vinculadas son transacciones mercantiles hacen referencia a personas jurídicas y entidades que estén interrelacionadas, es por ello que tienen los mismos socios o 
accionistas en más de una entidad e invierten su capital a fin de diversificar el riesgo y atraer más ingresos. No obstante, la compañía con mayor porcentaje de acciones tiene un poder de decisión que supera al de las demás (Losada, 2014). Para tal estudio se aplica este tipo de operaciones porque la empresa cuenta con más de 130 compañías relacionadas a nivel mundial e incluso más de $40 \%$ de sus operaciones están ligadas con la matriz ubicada en Hamburgo-Alemania. Los precios de transferencias analizados de manera administrativa obedecen a la responsabilidad que posee cada fracción del negocio (Horgen y Foster, 1991). Asimismo, para cumplir con las oportunidades de ventas solicitadas por los clientes es necesario el soporte de todas las áreas de la misma.

Por otra parte, en relación a los precios de transferencia comprenden el valor monetario de operaciones empresariales ya sean nacionales o internacionales de un mismo grupo institucional en donde cada una de ellas tiene obligaciones propias que afrontar, así como forjar el margen de ganancia individual (Diez de Castro, 1996). Dicho lo anterior, tales estudios evalúan las ventajas tributarias acorde a la economía de opción para realizar traspasos con valor económico entre las personas jurídicas o personas naturales que tengan vinculación directa o indirecta (Mejía, 1998).

La entidad en estudio se encuentra bajo el régimen general acorde al régimen tributario del Perú y ejecuta pagos de impuestos según a los tributos que está sujeto. El impuesto es una obligación tributaria para todos los ciudadanos que habitan en el Perú, el pago se realiza al momento que realizamos compras y por tal desembolso no recibimos retribución directa alguna por parte del gobierno (Villegas, 2019). También, cabe mencionar, que el impuesto hace referencia al aporte económico de las personas y empresas hacia el estado mediante la adquisición de algún bien o servicio por el cual no se tiene retribución directa con el individuo sino cubre las necesidades comunes de la sociedad (Flores, 1946). Finalmente, se sabe que las cargas tributarias comprenden las aportaciones que realizan los individuos ya sea como personas naturales o jurídicas y ello forma parte de la recaudación que realiza el gobierno (Rosas y Santillán, 1962).

La rentabilidad consiste en el retorno económico de lo invertido acompañado de un margen de ganancia el cual compensa la utilización del mismo. Uno de los propósitos fundamentales de las organizaciones consta en diseñar y conseguir el escenario que asegure los mejores resultados posibles en base al patrimonio empleado (Álvarez, Domínguez y Sánchez, 1989). Otro rasgo de la rentabilidad es la generación de ganancias en base a una inversión dineraria dada para un determinado periodo de tiempo (Vargas, 2016). Conviene subrayar que a rentabilidad refleja cómo se han gestionado las operaciones derivadas de las inversiones (Muñoz, 2008). Habría que decir también, que oda entidad busca obtener mayores beneficios económicos invirtiendo en menor medida su capital y bienes, con la finalidad de que en un determinado tiempo recupere más de lo invertido (Bravo, Lambreton y Márquez, 2007). Para concluir con la rentabilidad, está sujeta a contrastar la utilidad versus la inversión y con ello obtener el porcentaje de rentabilidad que indica si el negocio es factible acorde a las metas planteadas por la organización (Whelen y Hunger, 2007).

La empresa al aplicar los ratios de rentabilidad realiza un análisis más profundo respecto a la situación empresarial en la que se encuentra. Es por ello que, la rentabilidad económica es el 
margen de ganancia de los recursos que controla la empresa, así como la ganancia de una inversión en relación al capital invertido (Aguado, Chiquillanqui y Soca, 2017). Asimismo, la rentabilidad económica abarca la estructura productiva de los activos de la empresa independientemente de cómo se haya financiado (Sánchez, 2002). Respecto a la rentabilidad financiera necesita de la rentabilidad económica, de los recursos de la propia empresa más aún considera lo que le cuesta a la empresa obtener financiación de terceros (Corona, 2015).

Para refrendar los objetivos se consideraron tesis y artículos nacionales e internacionales, se revisó la publicación de (Lévano, 2018), en su tesis Impacto de las operaciones vinculadas en la gestión de una empresa inmobiliaria, Lima - 2018, y planteó como objetivo principal el análisis de las causas de las operaciones vinculadas en el ámbito contable de empresas del sector inmobiliario. También se revisó la tesis de investigación titulada Los precios de transferencia y su incidencia tributaria-financiera en las empresas vinculadas de fabricación de productos plásticos ubicadas en el distrito de Lurín, Lima-Año 2017 (Ramírez y Niño (2018). Por otro lado, el estudio de investigación de la tesis sustentada tiene por título Determinar si la gestión de ventas influye en la rentabilidad de las empresas del sector comercial del distrito de La Victoria - Lima, 2016 (Rojas, 2017). Más aún, se tuvo como investigación de tesis titulada Aplicación de los precios de transferencia entre empresas vinculadas y su incidencia en la determinación del impuesto a la renta caso: Transportes Kala S.A.C. Arequipa-2015 (Catacora, 2016). A su vez, en la tesis de investigación titulada Precios de transferencia entre empresas vinculadas y su incidencia en la gestión tributaria en empresas del grupo telefónica del Perú. En ella, ha considerado como objetivo principal demostrar si los precios de transferencia entre empresas vinculadas inciden en la gestión tributaria en empresas del Grupo Telefónica del Perú (Lather, 2013).

En el artículo de investigación que lleva por título El nuevo régimen de las operaciones vinculadas, tuvo como objetivo aterrizar las apreciaciones en la dimensión contable y provisionarlas acorde a la legislación española basado en los precios de transferencia entorno a lo internacional (Rosón,2018). Asimismo, la tesis denominada Diseño de estrategias para mejorar la rentabilidad de la empresa Produarroz S.A. Donde se tuvo por objetivo general diseñar estrategias para mejorar los costos y márgenes de rentabilidad de la compañía de la citada empresa (Romero, 2017). Mientras tanto, estudio titulado Propuesta para mejorar la rentabilidad en la Empresa Corpevin S.A. El principal objetivo de tal investigación consistió en evidenciar que se puede perfeccionar el rendimiento de la organización (Carpio y Díaz, 2016). Para finalizar, en la investigación titulada La producción y la rentabilidad de la empresa de construcciones y hormigones "Ecohormigones" Cía. Ltda., en el año 2010. Planteó como objetivo general el estudio del impacto de la producción hacia la rentabilidad de la organización indicada anteriormente (Andrade, 2011).

\section{Materiales y Métodos}

La investigación realizada tiene un enfoque de estudio cuantitativo, tuvo como finalidad describir la evolución e incidencias de las variables y con ello manifestar la descripción de las mismas. Asimismo, permite referir situaciones o fenómenos que se desea conocer (Hernández, Fernández y Baptista, 2014). Tal diseño, permitió estructurar, comparar la información con mayor relevancia y así sustentar los objetivos planteados. El tipo de estudio fue sustantiva. Las variables de estudio fueron las operaciones vinculadas y la rentabilidad de la empresa A. Hartrodt. La recolección de datos se obtuvo de los estados financieros de la entidad de tres 
periodos contables (Sánchez y Reyes 2006). Para el análisis cuantitativo se utilizó los ratios de rentabilidad, y los pagos por impuestos de IGV y renta de tercera categoría los cuales fueron procesados en el programa SPSS, cuyo manejo permitió describir la evolución e incidencia entre los indicadores, finalmente se aplicó el análisis vertical al estado de situación financiera y al estado de resultados integrales para describir la relevancia de los saldos de las partidas contables (Caballero, 2009).

\section{Resultados}

Los resultados cuantitativos se obtuvieron con la aplicación del método descriptivo a través del programa SPSS se tomó de la aplicación de ratios de rentabilidad realizado a los estados financieros y el consolidado de pago de tributos por periodo contable describiendo las incidencias y evoluciones de las operaciones vinculadas y la rentabilidad económica y financiera de la compañía, acorde a lo detallado en las siguientes interpretaciones:

\begin{tabular}{|c|c|c|c|c|c|c|c|c|c|c|c|}
\hline \multicolumn{9}{|c|}{ Ratio de rentabilidad económica } & \multirow{2}{*}{\multicolumn{3}{|c|}{$\begin{array}{c}\begin{array}{c}\text { Ratio de rentabilidad } \\
\text { financiera }\end{array} \\
\frac{\text { Rentabilidad Patrimonial }}{(\text { ROE })}\end{array}$}} \\
\hline$\frac{\text { Rentabilidad }}{\text { activos (R }}$ & & & Margen $B$ & ruto & & Margen & Neto & & & & \\
\hline $\begin{array}{c}\text { Utilidad Neta / } \\
\text { Pérdida }\end{array}$ & \multirow[t]{2}{*}{$=$} & \multirow[t]{2}{*}{$\%$} & $\begin{array}{c}\text { Utilidad Bruta / } \\
\text { Pérdida }\end{array}$ & \multirow[t]{2}{*}{$=$} & \multirow[t]{2}{*}{$\%$} & $\begin{array}{c}\text { Utilidad Neta } \\
\text { / Pérdida }\end{array}$ & \multirow[t]{2}{*}{$=$} & \multirow[t]{2}{*}{$\%$} & $\begin{array}{c}\text { Utilidad Neta } \\
\text { / Pérdida }\end{array}$ & \multirow[t]{2}{*}{$=$} & \multirow[t]{2}{*}{$\%$} \\
\hline Activos totales & & & Ventas netas & & & Ventas totales & & & Patrimonio & & \\
\hline
\end{tabular}

Ilustración \# 1 instrumento cuantitativo

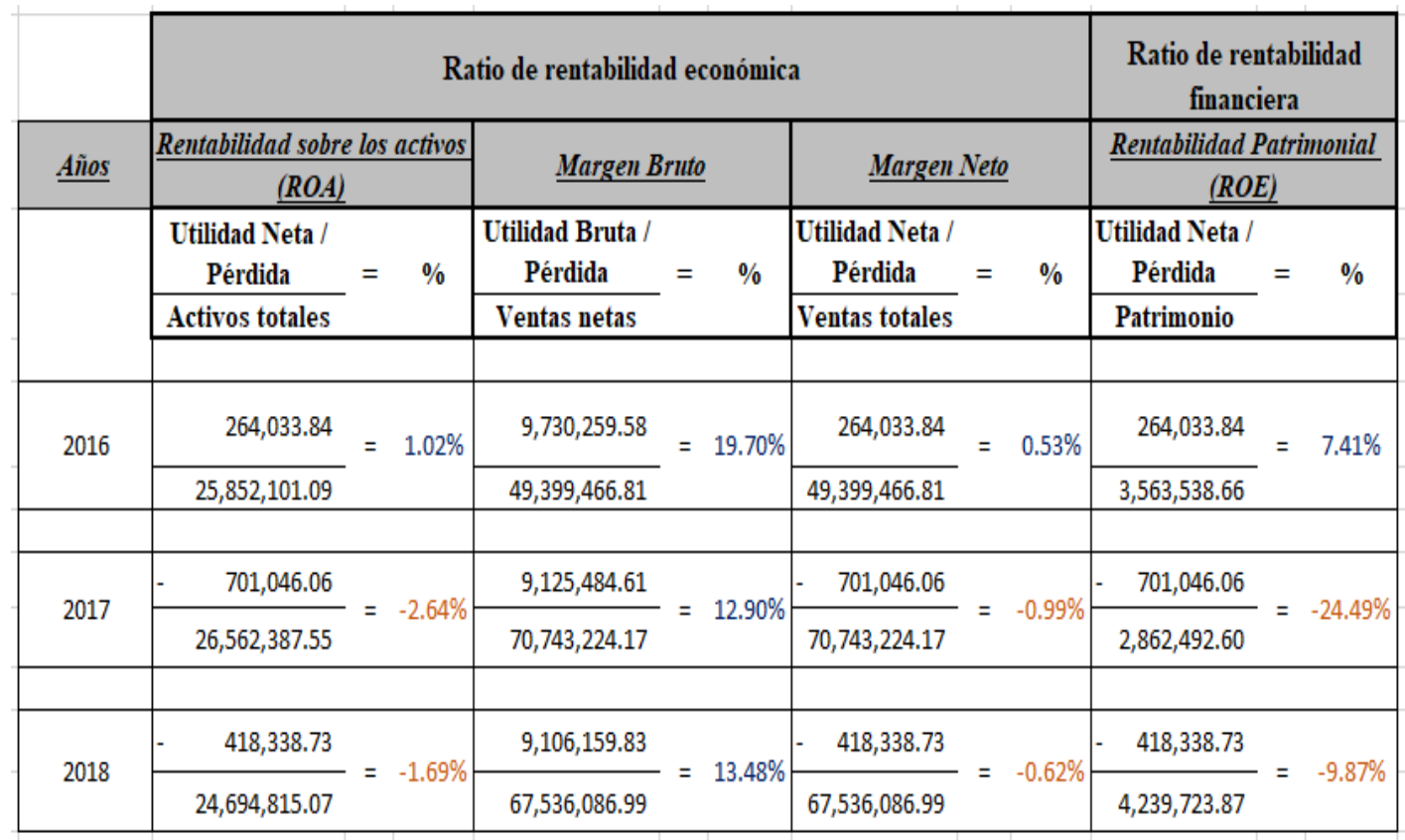

Ilustración \# 2 base de datos de ratios 


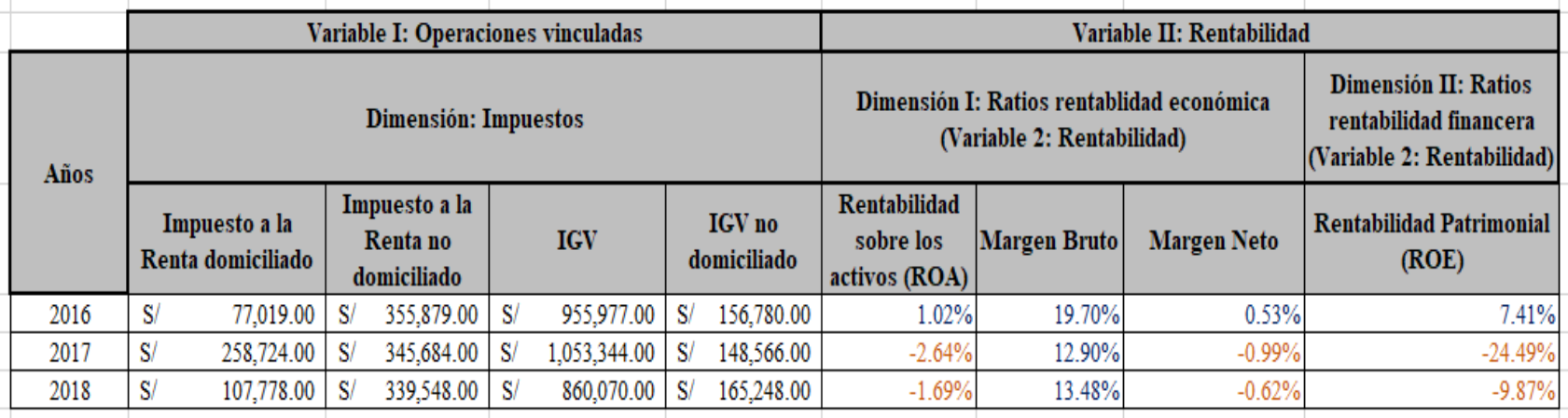

Ilustración \# 3 base de datos de pago de impuestos

El comportamiento del indicador denominado margen bruto, el cual está comprendido por la utilidad bruta sobre las ventas netas, señala el porcentaje de ganancia en relación a cada unidad monetaria vendida; todo ello se evalúa versus el impuesto a la renta tributado por las personerías jurídicas pertenecientes a un régimen tributario general que se tributa al fisco (SUNAT). Cabe resaltar en función a lo que se muestra en tal representación, que a menor pago de impuestos se muestra un incremento el margen de ganancia; respetando moderadamente los costos para llevar a cabo el servicio.

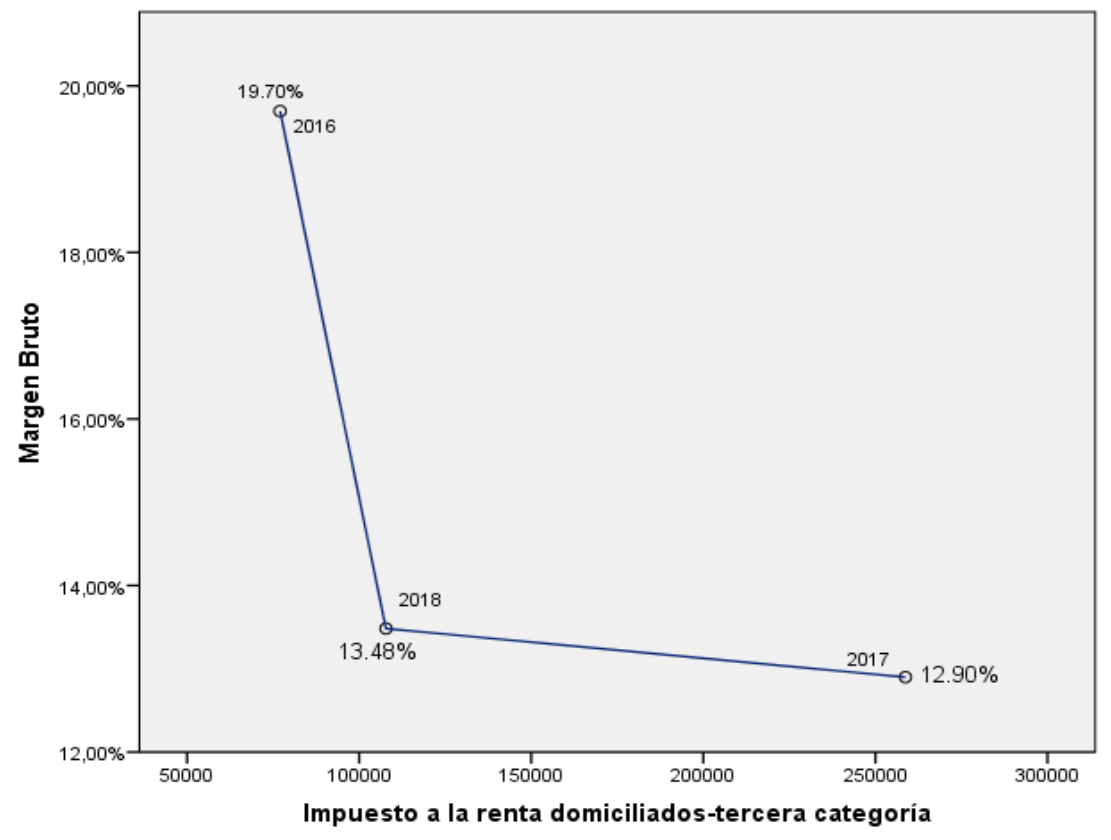

Figura \# 1 margen bruto e impuesto a la renta domiciliados tercera categoría

En este segundo esquema se evaluó el ratio de rentabilidad patrimonial más conocido como ROE por sus siglas en inglés "Return on Equity", tal razón financiera calcula el porcentaje de ganancia hacia los socios o propietarios; el cual está en función del resultado neto de un determinado periodo y esto se divide entre el patrimonio el cual está conformado por el capital social, reserva legal y por el mismo resultado obtenido del ejercicio. Dado el alcance anterior se puede observar que a menor pago de impuesto a la renta se incrementa el \% de rentabilidad. 


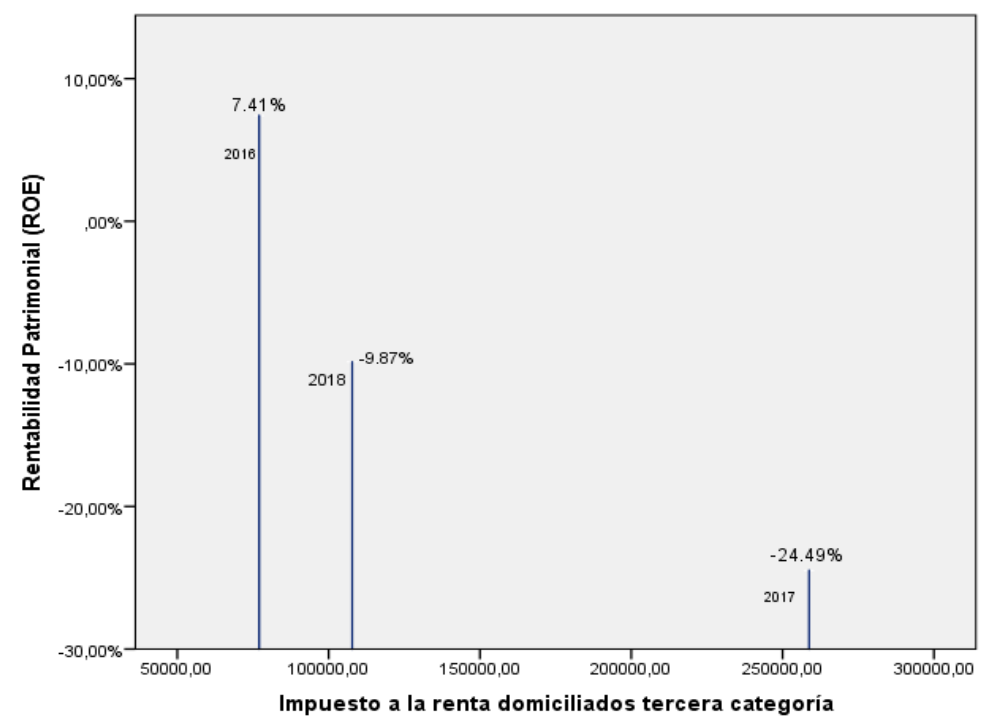

Figura \# 2 rentabilidad patrimonial e impuesto a la renta

En el siguiente gráfico se evalúa nuevamente el ratio "ROE" definido en el resultado anterior; y se logra observar que al realizar los pagos de impuestos generados al efectuar compras del exterior; también se ve afectado los años 2018 con $-9.87 \%$ y en mayor medida para el 2017 donde predomina un $-24.49 \%$ donde muestra un escenario preocupante dado que la capacidad de la entidad para que genere ganancias a los dueños no es favorable, tal razón financiera desfavorable también responde a que en el periodo 2017 se contaba con menor patrimonio debido a la pérdida neta del ejercicio. En el año 2016, se obtuvo un rendimiento de $7.41 \%$ a favor de los accionistas. Es necesario mencionar que, en el año 2018, los socios aportaron más capital y el resultado del ejercicio alcanzó a S/ -418,339.00, es por ello que no se tuvo tal magnitud de impacto negativo en el indicador a comparación con la razón financiera del periodo 2017 donde solo contaba con S/ 788,990 de capital y un resultado de S/ -701,046.00 y fue el periodo más afectado.

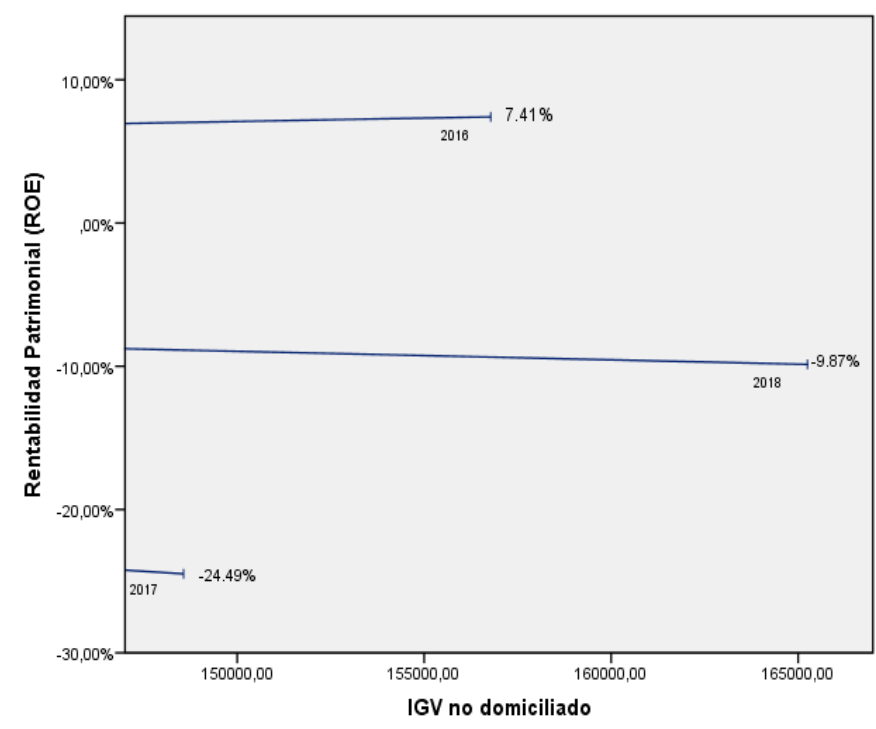

Figura \# 3 rentabilidad patrimonial e IGV 
Para la descripción del tercer esquema se muestra la fórmula del ratio margen neto que abarca la utilidad neta o pérdida sobre las ventas totales donde arroja el resultado y se analiza en porcentaje o en unidades monetarias y manifiesta el comportamiento de ventas a nivel nacional e internacional y su participación; para ello se deduce los costos y gastos que está en función a los desembolsos por renta con un $30 \%$ e IGV $18 \%$.

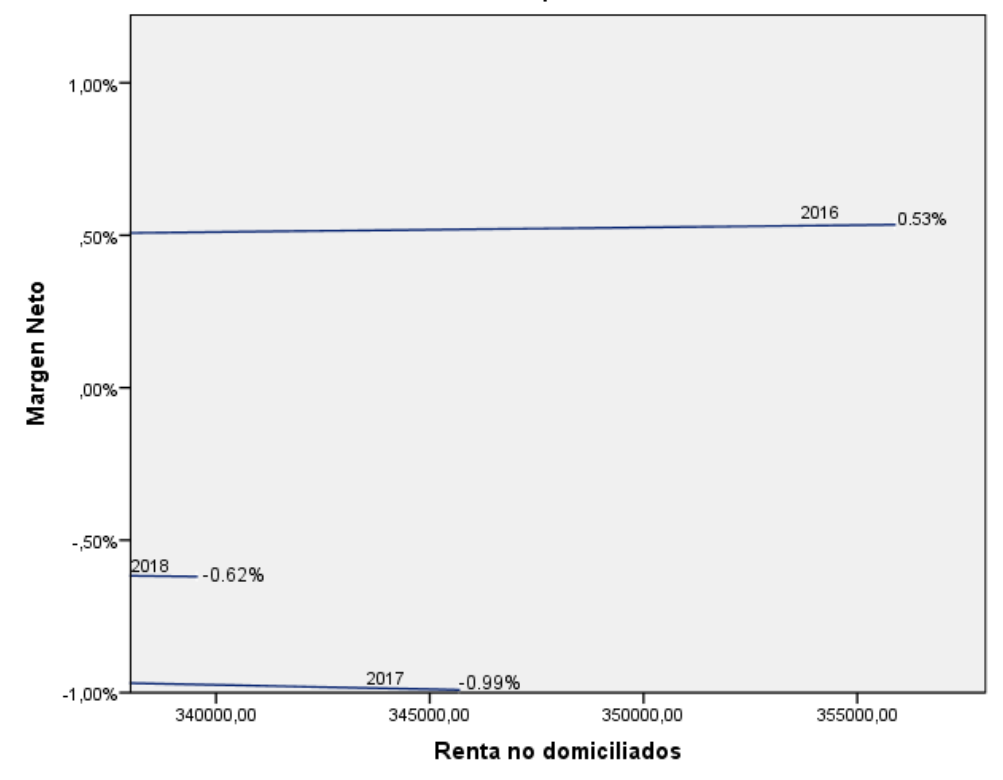

Figura \# 4 rentabilidad patrimonial e IGV

En el último cuadro se detallan los resultados referente a la rentabilidad sobre los activos conocido como "ROA" por sus siglas en inglés que quiere decir Return on assets la fórmula está comprendida por el resultado ya sea ganancia o pérdida sobre el total de activos de a un determinado periodo y se obtiene el porcentaje que demuestra el comportamiento de los recursos administrados y controlados por la entidad al evaluarlos en razón a lo tributado por renta de facturas o invoices emitidas por entidades no domiciliadas y sirve para concretar el servicio que se brinda a los clientes. En este caso al asociar tales dimensiones se muestra favorable el indicador cuando hubo mayor pago de renta no domiciliados, dado que para el 2016 está representado por el $1.02 \%,-2.64 \%$ para el periodo siguiente declinó en mayor medida dado al re resultado con mayor pérdida entre los tres ejercicios $y$, por último, constituido por $-1.69 \%$ para el periodo final. 


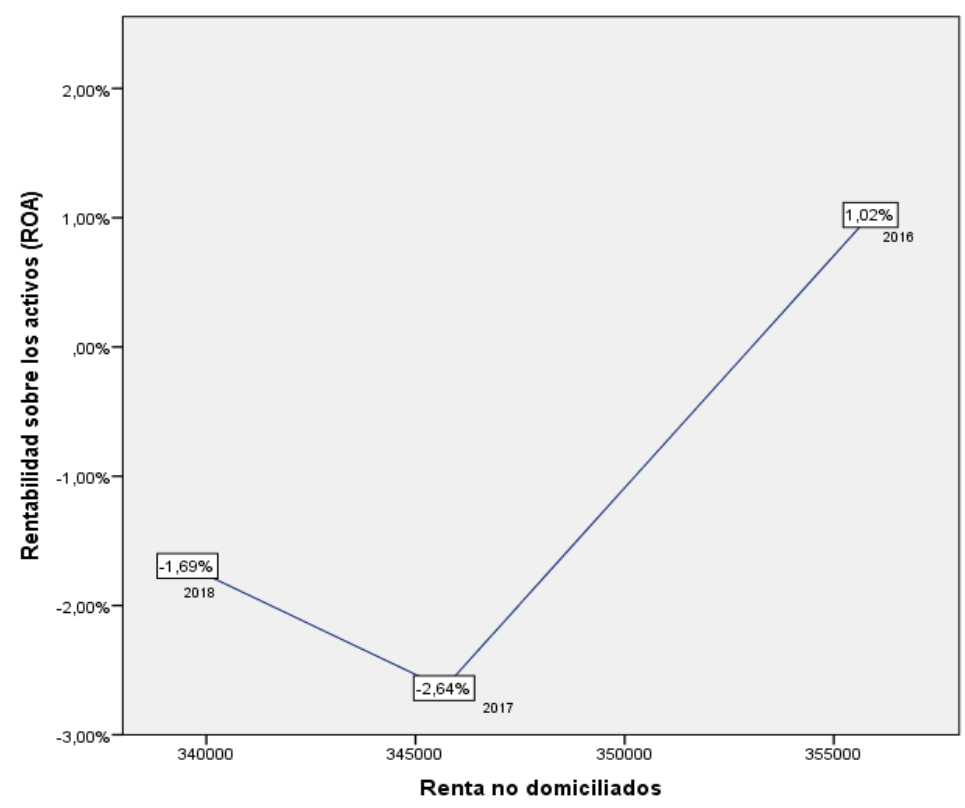

Figura \# 5 rentabilidad sobre los activos y renta no domiciliados

\section{Conclusiones}

Se analizó las operaciones vinculadas y la rentabilidad de la compañía, mediante el programa SPSS se realizó el análisis a los indicadores de los ratios y al pago de impuestos; donde se logró describir el nivel de incidencias entre tales dimensiones; donde los indicadores obtenidos nos muestran el comportamiento desde el año 2016 al 2018 respectivamente donde el ROA comprende de $1.02 \%,-2.64 \%$ y $1.69 \%$; respecto del margen bruto son los siguientes: $19.70 \%, 12.90 \%$ y $13.48 \%$; para el margen neto representado por $0.53 \%,-0.99 \%$ y $-0.62 \%$ y por último el indicador del ROE presentó el $7.41 \%,-24.49 \%$ y $-9.87 \%$.

Se analizó que las operaciones con vinculación económica a nivel intragrupo tienen incidencias en la rentabilidad de la empresa dado que los ratios de rentabilidad aplicados y además con el refuerzo del análisis vertical para los estados financieros que mostraron la relevancia de las partidas; en donde los costos de ventas y gastos operacionales manifiestan una gran participación, superior al $90 \%$ en función a los ingresos netos en los tres periodos consecutivos, estas razones demuestran el desempeño a nivel de ventas netas, de los costos de las operaciones y las incidencias con impuestos. Por otro lado, para el estado de situación financiera las cuentas contables más críticas del activo están comprendidas por las cuentas por cobrar representadas entre $57.57 \%$ al $61.80 \%$.

Se analizó que las operaciones vinculadas y la rentabilidad financiera alcanzan ciertas incidencias. La aplicación del ratio de rentabilidad respecto al beneficio sobre el patrimonio también se ven afectados por los elevados costos y gastos; ello indica que la compañía en los dos últimos periodos analizados no generó ganancias sino pérdidas a los propietarios. Asimismo, gracias al análisis vertical se tuvo en cuenta que Hartrodt ha incurrido en fuertes préstamos con partes vinculadas para que se puedan cubrir los costos del proceso operativo y estos alcanzan porcentajes desde el $27.55 \%, 31.66 \%$ hasta el $32.17 \%$ tales indicadores expresan que un tercio de 
la compañía está financiada por la casa matriz; ello generó obligaciones por intereses; es por esto que los gastos administrativos impactan negativamente en los resultados.

\section{Bibliografía}

Aguado, N., Chiquillanqui, G., \& Soca, Y. (Agosto de 2017). La planificación financiera y su influencia de la rentabilidad de la empresa Spectrum Ingenieros S.A.C. ubicada en Pueblo Libre. Lima, Lima, Lima: http://repositorio.ulasamericas.edu.pe/bitstream/handle/upa/230.

Álvarez, M., Dominguez, C., \& Sánchez, M. (1989). Rentabilidad y riesgo en el comportamiento financiero de la empresa. Madrid: Pérez Galdos, p. 22.

Andrade, C. (2011). La producciòn y rentabilidad de la empresa construcciones y hormigones "Ecohhormigones" Cía Ltda. en el año 2010. Ecuador.

Arias, J., Miranda, M., \& Villasís, M. (2016). Metodología de la investigación. Alergia México, 201-206.

Avantto, L. (2013). Precios de transferencia entre empresas vinculadas y su incidencia en la gestión tributaria en empresas del grupo telefónica del perú. Lima, Lima, Perú.

Bernal, C. (2006). Metodología de la Investigación. México: Pearson.

Caballero Bustamante. (2009). Definición y aplicación de los Ratios Financieros en las empresas. Lima.

Carpio, J., \& Díaz, Y. (2016). Propuesta para mejorar la rentabilidad en la Empresa Corpevin S.A. Guayaquil, Guayas, Ecuador.

Catacora, L. (2016). Aplicación de los precios de transferencia entre empresas vinculadas y su incidencia en la determinación del impuesto a la renta caso: Transportes Kala S.A.C. Arequipa-2015. Arequipa.

Chiavenato, I. (2006). Introducción a la teoría general de la administración. México: Mc Graw Hill Educación.

Corona, E., Bejarano, V., \& Gonzáles, J. (2014). Análisis de los estados financieros individuales y consolidados. España: p. 43.

Daft, R. L. (2011). Teoría y diseño Organizacional. México, D.F: Cengage Learning Editores, S.A. de C.V.

Datar, S., Horngren, C., \& Rajan, M. (1991). Contabilidad de Costos un enfoque gerencial. México: Pretince Hall Hispanoamericana S.A, Sexta Edición , p. 944.

Diez de Castro, E. (1996). Gestión de Precios. Madrid: Escuela Superior de Gestión Comercial y Marketing, p. 33.

D'Onofrio, P. (2008). Las teorías de la contabilidad socal y su relación con la responsablidad socal de la empresa. p. 33.

Fernández, J., Llorente, J., \& Pérez, G. (2008). Economía 1. Madrid: Editex.

Flores, E. (1946). Elementos de Finanzas Públicas Mexicanas. México D.F., p. 33.

Hernández, R., Fernández, C., \& Baptista, P. (2014). Metodología de la Investigación. México, México: McGraw W-Hill / Interamericana Editores, S.A. de C.V.

Hirache, L. (2015). Estados Financieros conforme a NIIF. Lima: Pacífico Editores S.A.C.

Junyent, F., \& Richard, E. (2009). Teoría jurídica., (pág. 25). Córdova. 
Lévano, A. (2018). Impacto de las operaciones vinculadas en la gestión de una empresa inmobiliaria, Lima - 2018. Lima, Lima, Perú.

Losada, M. (2014). ¿Qué son las operaciones vinculadas? p. 16.

Martínez, P. (20 de Julio de 2006). El método de estudio de caso: estrategia metodológica de la investigación científica. Pensamiento \& Gestión. Colombia.

Mejía, E. (1998). Precios de Transferencia. Revista nuevo consultorio fiscal. Número 202, p. 54.

Muñoz, J. (2008). Contabilidad Financiera. España: Pearson Educación, p. 421.

Organización de cooperación para el desarrollo económico. (2017). Transfer Pricing Guidelines for Multinational Enterprises. Francia.

Ramírez, L., \& Niño, M. (08 de Noviembre de 2018). Los precios de transferencia y su incidencia tributaria-financiera en las empresas vinculadas de fabricación de productos plásticos ubicadas en el distrito de Lurín, Lima-Año 2017. Lima, Lima, Perú.

Rojas, Z. (2017). Gestión de ventas y rentabilidad. Lima, Lima, Perú.

Romero, N. (Febrero de 2017). Diseño de estratégias para mejorar la rentabilidad de la empresa Produarroz S.A. Guayaquil, Guayas, Ecuador.

Rosas, A., \& Santillán, R. (1962). Teoría general de las finanzas públicas y el caso de Mèxico. México D.F: UNAM.

Rosón, P. (2011). El nuevo régimen de las operaciones vinculadas. España.

Rubio, M. (2014). El análisis documental: Indización y resumen en bases de datos especializadas. p. 1.

Rueda, J. (2017). Texto Único Ordenado de la Ley del Impuesto a la Renta. Lima: Editorial Gráfica Bernilla.

Sánchez, H., \& Reyes, C. (2006). Slide Player. Obtenido de Slide Player: https://slideplayer.es/slide/13164708/

Sánchez, J. (2002). Obtenido de http://ciberconta.unizar.es/leccion/anarenta/analisisR.pdf

Tilton, E. (1962). Teoría del crecimiento de la empresa. Madrid, España: Aguilar.

Vargas, J. (2016). Implementación de un sistema de costos por proceso y su efecto en la rentabilidad de la empresa Alpaca Color SA. Lima, Lima, Perú.

Villegas, H. (2019). SUNAT. Obtenido de SUNAT: http://www.sunat.gob.pe/institucional/quienessomos/sistematributario_entiende.html

Wheelen, T., \& Hunger, D. (2007). Administración estratégica y políticas de negocios. México: Pearson Educación, p. 263. 